\title{
Angle-Resolved Polarimetry of Antenna-Mediated Fluorescence
}

\author{
Abbas Mohtashami, Clara I. Osorio, and A. Femius Koenderink \\ Center for Nanophotonics, FOM Institute AMOLF, \\ Science Park 104, 1098 XG Amsterdam, The Netherlands \\ (Received 9 June 2015; revised manuscript received 18 September 2015; published 25 November 2015) \\ Optical phase-array antennas can be used to control not only the angular distribution but also the \\ polarization of fluorescence from quantum emitters. The emission pattern of the resulting system is \\ determined by the properties of the antenna, the properties of the emitters, and the strength of the antenna- \\ emitter coupling. Here we show that Fourier polarimetry can be used to characterize these three \\ contributions. To this end, we measure the angle- and Stokes-parameter-resolved emission of bullseye \\ plasmon antennas as well as spiral antennas excited by an ensemble of emitters. We estimate the average \\ antenna-emitter coupling on the basis of the degree of polarization and determine the effect of anisotropy in \\ the intrinsic emitter orientation on polarization of the resulting emission pattern. Our results provide not \\ only new insights into the behavior of bullseye and spiral antennas but also demonstrate the potential of \\ Fourier polarimetry when characterizing antenna-mediated fluorescence.
}

DOI: 10.1103/PhysRevApplied.4.054014

\section{INTRODUCTION}

Engineering the photonic environment of single emitters allows us to control their emission rate, angular distribution, and polarization. According to Fermi's golden rule, the emission rate depends on the local density of states, which can be manipulated with narrow-band devices, such as microcavities [1-3], or with broadband structures, which include plasmonic structures [4-6]. On the other hand, the angular distribution of emission from single emitters can be controlled by properly designed nanostructures that, after being excited by a pointlike emitter, act as a phased arrangement of coherent secondary sources [7]. This approach, which is analogous to phased-array engineering in radio frequency, has been demonstrated for single molecules coupled to scanning probe tips [8], plasmonic lattices [9-11], patch antennas [12,13], array antennas [14], and plasmonic bullseye antennas [15-21]. Moreover, with the advent of metasurfaces and metamaterials, it is evident that carefully shaped structures can simultaneously, although not independently, control the electric and magnetic components of the near field around an emitter $[22,23]$. Conversely, it has been shown that magnetoelectric scatterers can generate handed far-field emission when excited locally by an electric dipole source [24,25]. Plasmonic structures are, therefore, a promising way to simultaneously control the phase front and polarization of fluorescent sources.

An important consideration when using plasmonic structures for polarization control of fluorescence is that, in many experiments, fluorescence is only partially polarized. If an experiment uses a single and rigidly oriented dipole emitter, the emission will be fully polarized [14,25]. However, if the fluorescence is due to a randomly oriented ensemble of molecules, or if the molecular source is free to rapidly reorient from emission event to emission event, the emission will be at best partially polarized [26]. A relevant figure of merit for a plasmonic structure is, hence, in how far it is able to imprint polarization on an intrinsically poorly polarized source ensemble. This polarization transfer requires, on the one hand, a plasmon antenna with a strongly polarization-selective resonance and, on the other hand, a strong structure-emitter coupling, so that the polarized structure-mediated emission exceeds the direct emission of light into the far field that occurs for poorly coupled emitters. Here we show that by measuring the angle-resolved full polarization state of the emission, it is possible to separate direct emission from emission mediated by a photonic structure and, therefore, to estimate the average antenna-emitter coupling. We use a $k$-space polarimeter that combines a Fourier microscope with a polarimeter [25,27-29] to retrieve the Stokes parameters $\left(S_{0}, S_{1}\right.$, $S_{2}$, and $S_{3}$ ) of fluorescence emitted in the vicinity of bullseye antennas and spirals [19-21,30]. The Stokes parameters allow us to calculate the ratio of polarized to unpolarized light, to separate those contributions to the total intensity, and to calculate the electric-field components that describe the polarized part [31-33].

\section{EXPERIMENTAL METHODS}

\section{A. Setup}

Figure 1(a) shows our experimental setup composed of a fluorescence microscope equipped with an optional Fourier-transforming lens $\left(f_{\text {Fourier }}=200 \mathrm{~mm}\right)$ and a rotating-plate polarimeter. As an excitation light source, we use a broadband supercontinuum laser (Fianium) filtered by an acousto-optical tunable filter and a bandpass filter $(680 \pm 10 \mathrm{~nm})$. The polarization of the excitation is set to 
horizontal or right-handed circular using a linear polarizer and a quarter-wave plate. A $10 \times$ objective $(\mathrm{NA}=0.25$, Nikon Plan) focuses the light on the sample, where it serves to pump near-infrared fluorophores diffusing around the sample. A $60 \times$ objective $(\mathrm{NA}=0.7$, Nikon CFI Plan Fluor) collects the fluorescence and directs it to a spatial filter. The spatial filter is composed of a 1:1 telescope (two lenses of $f_{\text {telescope }}=50 \mathrm{~mm}$ ) and a 300- $\mu \mathrm{m}$ pinhole, which selects an area of about $20 \mu \mathrm{m}$ across in the sample plane. The excitation light and the fluorescence are separated by a 690-nm long-pass filter. A section of the broad fluorescence spectrum is selected by a bandpass filter centered at $750 \mathrm{~nm}$ with a full width at half maximum of $40 \mathrm{~nm}$. Finally, a polarimeter consisting of a quarter-wave plate and a linear polarizer is placed before the $f=200 \mathrm{~mm}$ tube lens and the silicon CCD camera (Photometrics CoolSNAP EZ). The exposure time of the camera varies between 40 and $60 \mathrm{~s}$ depending on the structure, with a constant pump power of $10 \mu \mathrm{W}$ to avoid saturation, which will modify the way the dye inherits polarization anisotropy from the pump beam.

\section{B. Samples}

We fabricate cylindrical and spiral bullseye antenna samples from a 200-nm-thick layer of gold evaporated on top of a glass coverslip covered by a 5-nm-thick chromium adhesion layer. Using a focused ion beam, we mill a 200-nm-diameter hole through the gold layer and approximately 50-nm-deep trenches concentric to the hole. The grooves have a 50\% duty cycle. We study bullseye antennas as well as anticlockwise and clockwise Archimedean spiral antennas [Figs. 1(c), 1(d), and 1(e), respectively]. For each structure, the distance between the center and the first groove is $a=330 \mathrm{~nm}$ and between consecutive grooves is $p=600 \mathrm{~nm}$. For reference, we also fabricate single holes with no structures around. On top of the structures, we mount a flow cell containing Alexa Fluor 700 dye molecules dissolved in water at a concentration of $10 \mu \mathrm{M}$, which have an emission peaked at $723 \mathrm{~nm}$ with a full width at half maximum of about $50 \mathrm{~nm}$. On the basis of scattering measurements [29], we anticipate the bullseye antennas to imprint directionality in the form of a narrow doughnut beam on fluorescence emitted by molecules in the central aperture $[15,20,34]$.

\section{Polarimetry}

In order to determine the polarization state of the light emitted by dye molecules in the vicinity of the nanoantennas, we retrieve the angle-resolved Stokes parameters $S_{0}, S_{1}, S_{2}$, and $S_{3}$. This procedure requires measuring Fourier images of the fluorescence intensity $I_{n}$ transmitted by the polarimeter when performing as a linear polarizer (horizontal, vertical, $45^{\circ}$ and $135^{\circ}$, respectively) and as a circular polarizer (right and left handed). The Stokes parameter $S_{0}$ equal to the total intensity of the fluorescence
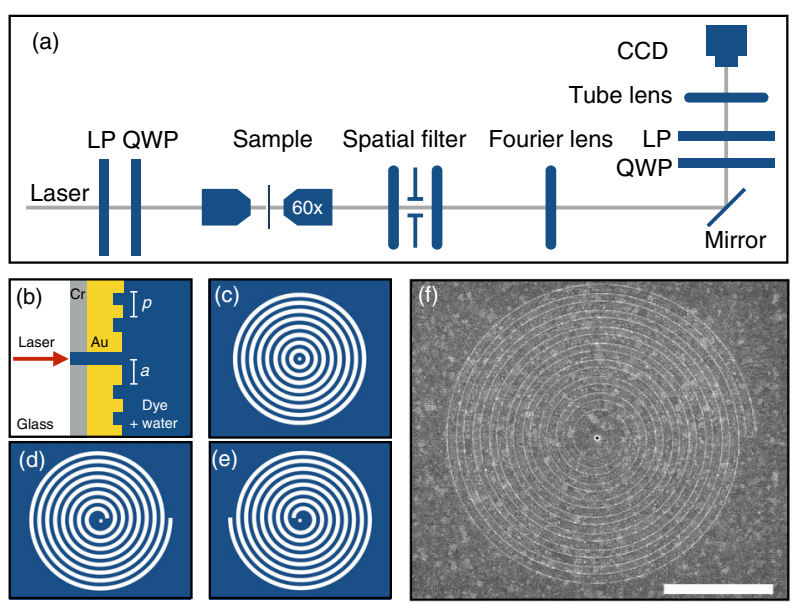

FIG. 1. (a) The experimental setup is based on a fluorescence Fourier microscope. The polarization of the incident light is set by a linear polarizer (LP) and a quarter-wave plate (QWP), after which the light is focused into the sample. The resulting fluorescence is then collected by a $60 \times$ microscope objective $(\mathrm{NA}=0.7)$; a spatial filter allows us to select particular regions in the sample plane. The Fourier and tube lenses are placed such that the back focal plane of the objective is imaged into the camera sensor. Finally, as a polarimeter, we use a quarter-wave plate and a linear polarizer. (b) Crosscut of the sample. The dye molecules diffuse freely on top of each sample in a water solution contained by a flow cell. Sketches of the fabricated (c) bullseye, (d) anticlockwise-spiral, and (e) clockwise-spiral plasmonic nanoantennas. (f) Scanning electron micrograph shows the fabricated clockwise-spiral plasmonic nanoantenna. The scale bar corresponds to $4 \mu \mathrm{m}$.

is given by the sum of any pair of orthogonally polarized intensities (e.g., $S_{0}=I_{H}+I_{V}$ ). On the other hand, the difference between each pair of orthogonally polarized intensities determines the other Stokes parameters $\left(S_{1}=I_{H}-I_{V}, S_{2}=I_{45}-I_{135}\right.$, and $\left.S_{3}=I_{\mathrm{RHC}}-I_{\mathrm{LHC}}\right)$ [31]. When normalized to the total intensity, these last three Stokes parameters take values between -1 and 1 . The two extreme values correspond to an emission fully polarized in one of the two orthogonal polarizations used to define the parameter, while 0 corresponds to the case where both polarizations contribute equally to the total emission.

\section{Calibration of dye properties}

In addition to considering their interaction with the antennas, other properties of the dye must be accounted for when interpreting plasmon-mediated radiation patterns. We measure the fluorescence lifetime $\tau_{f}$ and the rotational diffusion $\tau_{r}$ of the Alexa Fluor 700 molecules using timeresolved fluorescence-intensity measurements $[35,36]$ in an experimental setup described in detail in Ref. [37]. By moving to an area of the sample where the glass substrate is not covered by chromium or gold, we measure the fluorescence emission in transmission through a glass dye-in-water system. After exciting the molecules with 

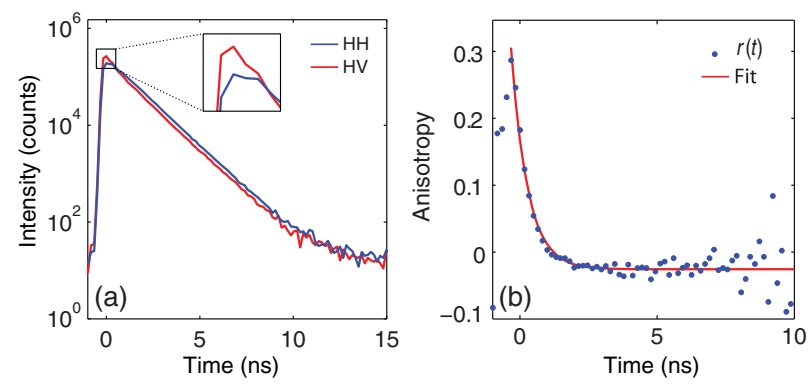

FIG. 2. After exciting the molecules with horizontally polarized light, we measure the horizontally $(\mathrm{HH})$ and vertically $(\mathrm{HV})$ polarized time-resolved fluorescence intensities (a). The inset shows the region where the two time traces differ the most. These measurements are used in the calculation of the time-resolved anisotropy (b), which is fitted to a single exponential.

horizontally polarized light and without using polarizers in the detection, we measure the fluorescence lifetime of the dye $\tau_{f}=1 \pm 0.05 \mathrm{~ns}$. Next, we measure separately the coand cross-polarized components of the fluorescence decay trace [Fig. 2(a)] and extract the time-resolved fluorescence anisotropy $r(t)$ [Fig. 2(b)], which is given by

$$
r(t)=-\frac{i_{\mathrm{HH}}(t)-G i_{\mathrm{HV}}(t)}{i_{\mathrm{HH}}(t)+2 G i_{\mathrm{HV}}(t)} .
$$

Here, $i_{X Y}(t)$ denotes the $Y$-polarized component of the fluorescence decay trace obtained with $X$-polarized excitation, and the factor $G=\int i_{\mathrm{VH}}(t) d t / \int i_{\mathrm{VV}}(t) d t$ compensates for any polarization bias of the detection system. Fitting the anisotropy to a single exponential yields a value for the rotational diffusion time of the molecules of $t_{r}=0.6 \pm 0.05 \mathrm{~ns}$, just slightly shorter than the lifetime. These measurements are evidence that, indeed, rotational averaging is slow compared to the fluorescence lifetime, and, therefore, the molecules retain significant polarization memory. Notice that, thanks to the Purcell enhancement (expected to be approximately equal to 4 according to Ref. [34]) close to the structure, the emission will become faster, and the molecules will become more "rotationally frozen." Following Refs. [35,36], we use the retrieved time constants to calculate the steady-state anisotropy as a function of the angle between the excitation and emission dipoles of the molecule. The maximum value for the steadystate anisotropy corresponding to aligned dipole moments is $r=0.15$, which predicts a maximum value of $S_{1} / S_{0}=0.21$.

To measure the complete polarization state of the light emitted by the dye far from any structure, we use Fourier polarimetry. The normalized Stokes parameters $S_{1} / S_{0}$, $S_{2} / S_{0}$, and $S_{3} / S_{0}$ of the fluorescence show constant values throughout $k$ space, with values that depend on incident polarization. For incident circularly polarized light, the angular average of each of these parameters is close to zero $\left(\left\langle S_{1} / S_{0}\right\rangle=1.3 \times 10^{-2},\left\langle S_{2} / S_{0}\right\rangle=0.1 \times 10^{-2}\right.$, and $\left\langle S_{3} / S_{0}\right\rangle=0.1 \times 10^{-2}$ ) indicating unpolarized emission, as expected for an isotropic ensemble of emitters. On the other hand, when excited by horizontal polarized light, the emission is biased towards the incident polarization, with an angular average of the first Stokes parameter $\left\langle S_{1} / S_{0}\right\rangle=0.23$ (while $\left\langle S_{2} / S_{0}\right\rangle=1.2 \times 10^{-4}$ and $\left\langle S_{3} / S_{0}\right\rangle=3.1 \times 10^{-2}$ ). These values are consistent with those obtained from the measured rotational diffusion time and fluorescence decay and confirm the large fluorescence anisotropy of the Alexa Fluor 700 molecules that we use.

Choosing a dye system with a fluorescence lifetime comparable to the rotational diffusion has several advantages. The dye is almost rotationally frozen, with or without the Purcell enhancement of the structure, which simplifies the analysis of the radiation patterns. In addition, we can probe our plasmonic system in two distinct ways due to the anisotropy. On one hand, we can drive the antennas with a completely unpolarized ensemble of emitters by applying circular input polarization. On the other hand, we can drive the system with a preferentially linearly polarized ensemble of emitters by pumping with a linear input polarization. As we will show, this results in completely different polarization emission patterns. A third class of experiments with fully oriented driving can be achieved only with single molecules or physically aligned dipoles. Finally, while translational diffusion during a fluorescence cycle is negligible (subnanometer), given the acquisition time is $100 \mathrm{~s}$ and the approximately $10 \mu \mathrm{s}$ that it takes for a dye molecule to diffuse into and out of the central hole, we average over essentially all possible locations of the dye molecules in the hole.

\section{BULLSEYE MEASUREMENTS}

We examine the angle-resolved polarization state of the light generated by molecules in a single nanoaperture and in a bullseye structure. Figure 3 shows the retrieved Stokes parameters for fluorescence emitted when the structures are illuminated with circularly [Figs. 3(a) and 3(c)] and linearly polarized light [Figs. 3(b) and 3(d)]. Single-hole apertures in Figs. 3(a) and 3(b) show an angularly isotropic emission pattern $S_{0}$ independent of the incident polarization [20,34]. The other Stokes parameters and, therefore, the polarization, also show little angular structure. There is a small polarization bias [see, for instance, $S_{1}$ and $S_{2}$ in Fig. 3(a)] due the polarizing effects of optical elements placed before the polarimeter, such as the high-NA-microscope objective and a silver mirror. Despite the fact that the nanoaperture modifies the polarization of the incident field, horizontally polarized excitation still increases the emission of horizontally polarized photons, as shown by the increase of the angular average of $S_{1}$ from $\left\langle S_{1} / S_{0}\right\rangle=3.0 \times 10^{-2}$ for circularly polarized excitation to $\left\langle S_{1} / S_{0}\right\rangle=0.15$ for linearly polarized excitation.

In contrast to single holes, bullseye structures show very directional emission patterns $S_{0}$, which result from far-field interference of direct emission and emission that is first 

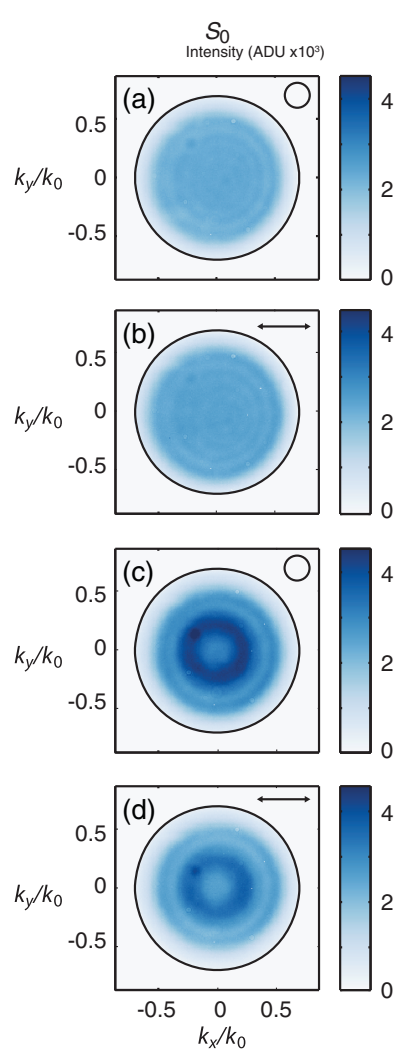

$S_{1} / S_{0}$

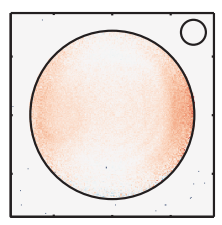

$S_{2} / S_{0}$
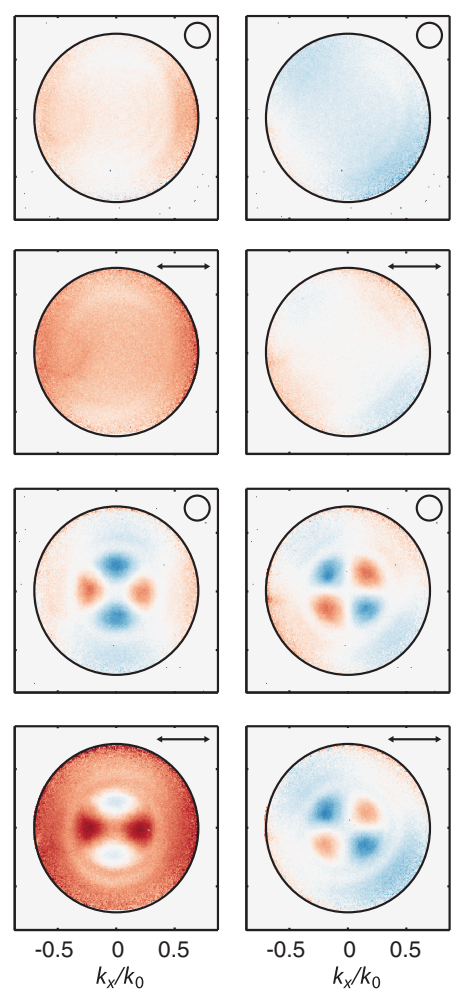
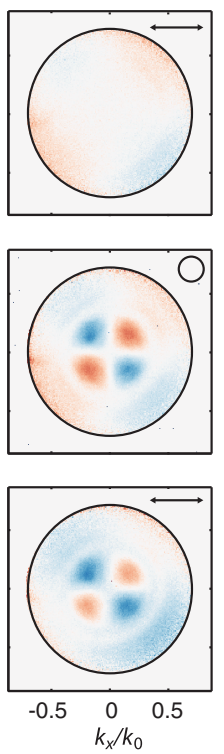

$S_{3} / S_{0}$

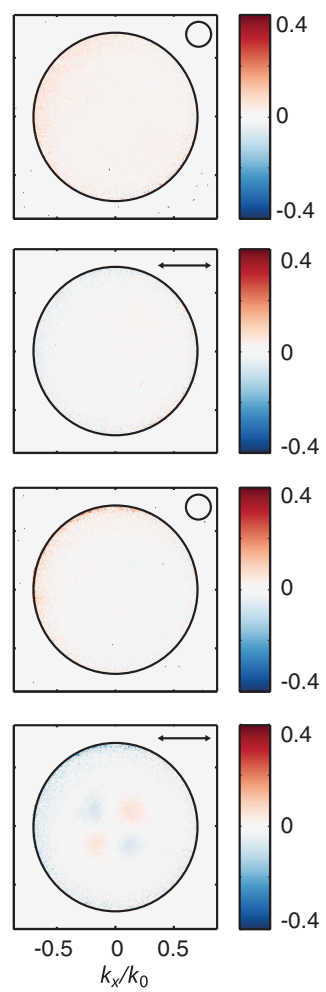

FIG. 3. $k$-space distribution of retrieved Stokes parameters $S_{0}$ and $S_{1} / S_{0}, \quad S_{2} / S_{0}$, and $S_{3} / S_{0}$ for the emission of a single-hole aperture (a),(b) and a bullseye (c),(d) excited with circularly (a),(c) and linearly polarized light (b),(d) symbolized by a circle or an arrow in each image. In all cases, the detection wavelength is $750 \pm 20 \mathrm{~nm}$. Images are clipped at the NA of our objective (black circle at 0.7 ). funneled into plasmons and subsequently outcouples at the antenna grooves $[20,21]$. These features are slightly blurred by the spectral collection bandwidth [(750 \pm 20$)$-nm filter] and are also limited intrinsically in sharpness by the size of the antenna (radius of approximately ten wavelengths in water). The clear structure in the Stokes parameters $S_{1}$ and $S_{2}$ in Figs. 3(c) and 3(d) indicates two important features of the polarization state of the emitted light. First, even when using circularly polarized excitation to pump a random isotropic ensemble of molecules, there are angular regions where $S_{1} / S_{0}$ and $S_{2} / S_{0}$ are nonzero. Therefore, the emitted light is at least partially polarized due to an interaction with the plasmon antenna. Second, the cloverleaf pattern with alternating signs in $S_{1}$ and $S_{2}$ suggest that the imparted polarization is largely radial, as expected, due to the role of plasmons that, after being excited by the molecules, propagate radially from the central hole to the grooves and, subsequently, scatter out in a narrow doughnut by diffraction at the grooves. Since plasmons are TM waves [38,39], one expects the outcoupled doughnut to have a welldefined radial polarization. Additionally, for all angles of emission, $S_{3} / S_{0} \approx 0$, with the exception of very faint features in Fig. 3(d), which we attribute to the presence of a mirror in front of the polarimeter. This means that the measured light shows no circularly polarized component since both contributions to the emission, i.e., direct emission by the molecules and plasmon scattering from the grooves, are either linearly polarized or unpolarized. In contrast, if one performs scattering experiments on bullseyes [29], linearly polarized input light can be scattered as circularly polarized light at non-normal angles. This is possible since the scattering includes a coherent superposition of the diffracted pump beam and light scattered by the bullseye groove [40].

Next, we convert measured Stokes parameters into the degree of polarization of the emitted light, i.e., the ratio of polarized light to total intensity, as well as the degrees of linear (DLP) and circular (DCP) polarization according to

$$
\begin{aligned}
\mathrm{DP} & =\frac{\sqrt{S_{1}^{2}+S_{2}^{2}+S_{3}^{2}}}{S_{0}}, \\
\mathrm{DLP} & =\frac{\sqrt{S_{1}^{2}+S_{2}^{2}}}{S_{0}}, \\
\mathrm{DCP} & =\frac{\left|S_{3}\right|}{S_{0}} .
\end{aligned}
$$

Figure 4 shows these three quantities as well as the total emission $S_{0}$ for light emitted by fluorophores in the vicinity of the bullseye antenna when excited with circularly [Fig. 4(a)] and linearly polarized light [Fig. 4(b)]. In all instances, $\mathrm{DCP} \approx 0$ is negligible, while the DLP is, within error, identical to the DP. The figure shows that the DP depends on the incident polarization, with maximum values going from $\mathrm{DP}_{\max }=0.25$ (with a mean value over the NA of $\mathrm{DP}_{\text {mean }}=0.08$ ) for circularly polarized excitation to $\mathrm{DP}_{\text {max }}=0.39$ (with $\mathrm{DP}_{\text {mean }}=0.21$ ) for linear polarization. These values of DP result from an ensemble 

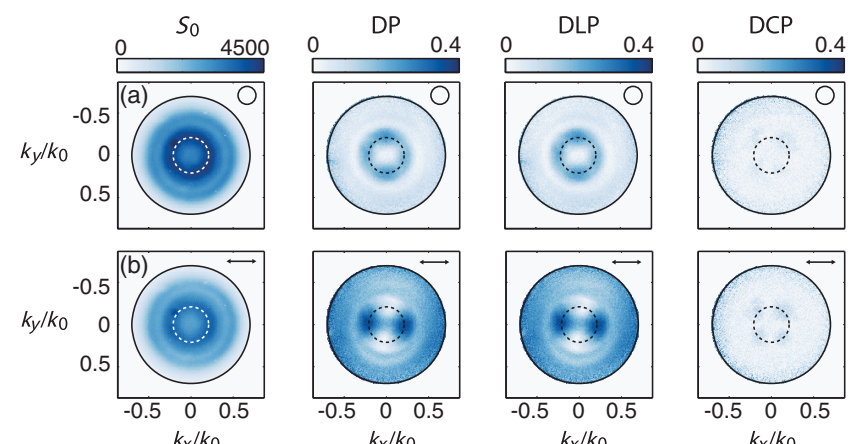

$k_{x} / k_{0}$

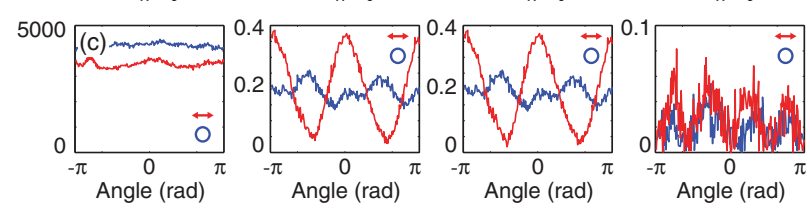

FIG. 4. Emission pattern $\left(S_{0}\right)$, total degree of polarization (DP), degree of linear polarization (DLP), and degree of circular polarization (DCP) for a bullseye structure illuminated by circularly polarized (a) and linearly polarized light (b). The crosscuts in (c) compare each of the quantities at $|\mathbf{k}| /\left|k_{0}\right|=0.2$ [marked with a dotted circle in (a) and (b)] for circularly (blue) and linearly (red) polarized excitation. In all cases, the detection wavelength is $750 \pm 20 \mathrm{~nm}$.

average and are set by the fraction of light emitted with the mediation of the plasmon resonance. Given the information obtained during the dye calibration, we can establish that in the case of circularly polarized excitation, $\mathrm{DP}_{\max }$ is a direct measurement of the percentage of light emitted into the far field via the antenna, while for incident linear polarization, $\mathrm{DP}_{\max }$ sets a upper bound, given that direct emission is partially polarized.

The incident pump polarization influences not only the maximum attained degree of polarization $\mathrm{DP}_{\max }$ but also the angular distribution of the different degrees of polarization. Figure 4(c) shows $S_{0}$, DP, DLP, and DCP as a function of the polar angle for a fixed ratio $|\mathbf{k}| /\left|k_{0}\right|=0.2$ (i.e., at the doughnut beam opening angle of approximately $9^{\circ}$ in water) indicated by the dotted circles in Figs. 4(a) and 4(b). While circularly polarized excitation (blue) produces a ring of emission that is approximately uniformly polarized, linearly polarized excitation (red) results in a doughnut beam that has strongly polarized and unpolarized lobes. These patterns result from the incoherent superposition of the homogeneous and (slightly) horizontally biased emission of molecules not coupled to the antenna and the radially polarized donut beam generated by the plasmon scattering. While these two contributions have the same polarization around $\left|k_{y}\right| /\left|k_{0}\right|=0$ effectively increasing DP, around $\left|k_{x}\right| /\left|k_{0}\right|=0$, the radially polarized light and the background are orthogonally polarized decreasing DP, thereby causing the "unpolarized" lobes.

It is possible to obtain further information about the structure of the emission patterns by separating polarized from unpolarized emission and using other figures of merit to describe the polarized part. The amplitude of the electricfield components in Cartesian coordinates $\left|E_{x}\right|,\left|E_{y}\right|$, and the phase between them, $\delta$, are given by [31]

$$
\begin{aligned}
\left|E_{x}\right|^{2} & =\left(S_{0}+S_{1}\right) / 2, \\
\left|E_{y}\right|^{2} & =\left(S_{0}-S_{1}\right) / 2, \\
\delta & =\arg \left(S_{2}+i S_{3}\right) .
\end{aligned}
$$

Transforming these Cartesian back-aperture field components to cylindrical coordinates provides direct access to the $p$ - and $s$-polarized components of the spherical wave emitted by the object, as evident from the "Abbe sine condition" transformation rules by which objectives transform a spherical wave from an object point to a cylindrical collimated beam [38]. Figure 5 shows the radial $I_{p}=\left|E_{r}\right|^{2}$ and tangential $I_{s}=\left|E_{\varphi}\right|^{2}$ intensity distributions for the polarized part of the fluorescence generated at our bullseye antenna. Under circularly polarized excitation, the molecules' direct emission is mostly unpolarized. Therefore, Fig. 5(a) shows the fully $p$-polarized intensity resulting from the scattering of radially propagating plasmons by the grooves $[15,19,20]$. On the other hand, the direct emission from oriented molecules appears as tangentially polarized at angles where the tangential direction coincides with the direction of the incident polarization and where the radially polarized emission from the structure does not cancel the effect; Fig. 5(b). This behavior is confirmed when retrieving other figures of merit that describe the polarized part of the emission, such as the parameters of the polarization ellipse shown in the two last columns of Fig. 5.

While bullseye-mediated emission results in a linearly (radially) polarized doughnut beam, one expects handed structures to impose handed polarization. Figure 6 shows the angle-resolved Stokes parameters of light emitted in the vicinity of an anticlockwise [Fig. 6(a)] and clockwise [Fig. 6(b)] Archimedean spiral. As excitation, we use circularly polarized pump light, which should again result
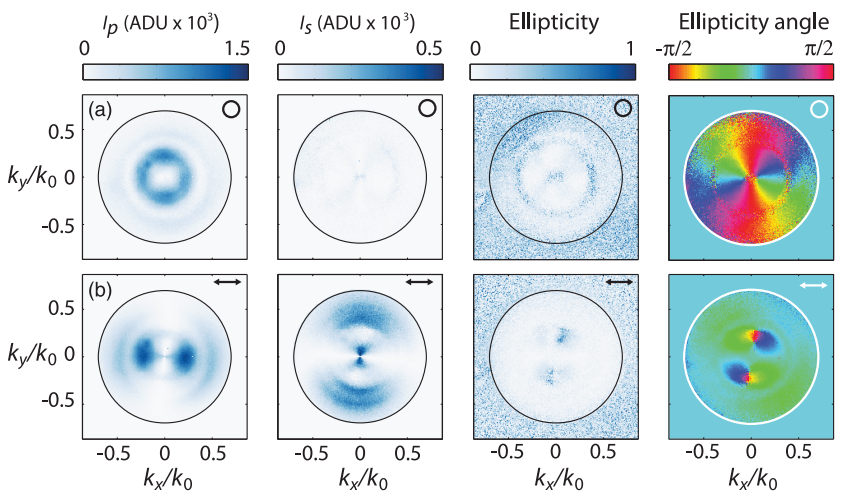

FIG. 5. Intensities of $p$ - and $s$-polarized field components, ellipticity, and ellipticity angle of the molecular fluorescence generated when a bullseye structure is illuminated by circularly (a) and linearly (b) polarized light. 


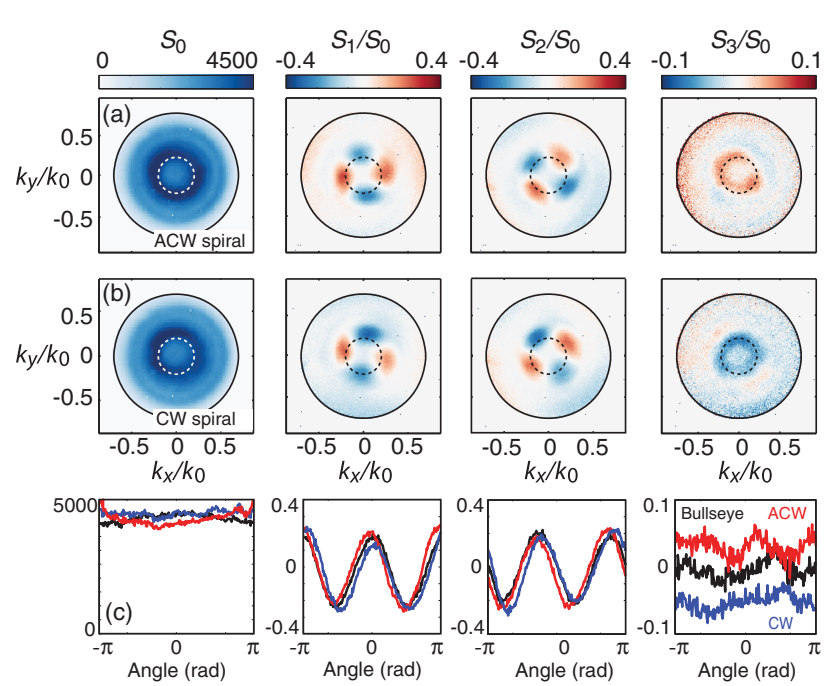

FIG. 6. Angle-resolved Stokes parameters $S_{0}, S_{1} / S_{0}, S_{2} / S_{0}$, and $S_{3} / S_{0}$ of an (a) anticlockwise spiral (ACW) and (b) clockwise (CW) Archimedean spiral excited with circularly polarized light. For comparison, the crosscuts in (c) include the Stokes parameters of the bullseye under the same illumination (black lines).

in excitation of a random isotropic ensemble of dipole moments. While the total intensity distribution $S_{0}$ resembles closely the bullseye emission, the handedness of the structures is translated into the other Stokes parameters. The cloverleaf patterns observed in $S_{1} / S_{0}$ and $S_{2} / S_{2}$ for bullseye emission change shape and orientation resulting in nonmirror symmetric angular distributions. Crosscuts at $|\mathbf{k}| /\left|k_{0}\right|=0.2$ show that the asymmetry reverses with reversal of the spiral handedness. In addition, the $S_{3} / S_{0}$ patterns show a small amount of circularly polarized light emitted by the structures in the regions of higher total emission with a handedness given by the handedness of the structure. The crosscut coincides with the region of maximum circularly polarized emission which, however, accounts only for $4 \%$ of the total emission or, equivalently, $18 \%$ of the polarized emission. Increasing this fraction separates into two challenges: on the one hand, it requires improving the overall coupling strength between emitters and antenna (raising DP) and on the other hand, providing stronger chirality to enhance DCP and DP, as has been shown is the case for quantum dots coupled to split-ring resonators [25]. From a methods point of view, the data set shows the large potential of Fourier polarimetry to determine the polarization performance of single-plasmon antennas coupled to fluorophores.

\section{CONCLUSIONS}

We report Fourier polarimetry measurements on the emission of bullseye and spiral antennas coupled to an ensemble of Alexa Fluor 700 dye molecules dissolved in water. The measured angle-resolved Stokes parameters allow us to separate polarized from unpolarized contributions to the fluorescence, providing deeper insight into the behavior of both the emitters and the antenna and their coupling. In the particular dye system we choose, fluorescence decay is fast, almost on the time scale of rotational diffusion. Thereby, we can probe both the case of a "frozen" anisotropic molecular ensemble using linear excitation polarization and a random orientational ensemble of emitters using circularly polarized excitation. In the latter case, the polarized part of the emission is only due to the scattering from the bullseye and has a radially polarized emission pattern. In this case, the degree of polarization directly gives the ratio of light emitted via the antenna to light emitted in total. In contrast, under linearly polarized excitation, a very different angular distribution of polarized and unpolarized emission is observed, showing the importance in plasmonfluorescence-enhancement measurements to have a good grasp of the source orientation distribution. Finally, we demonstrate a small amount of circularly polarized emission when using Archimedean spirals, which can likely be enhanced by increasing the chirality of the antenna and its coupling to the emitters, as shown in Ref. [25].

\section{ACKNOWLEDGMENTS}

This work is part of the research program of the Foundation for Fundamental Research on Matter (FOM), which is part of the Netherlands Organization for Scientific Research (NWO). This work is supported by NanoNextNL, a micro- and nanotechnology consortium of the Government of The Netherlands and 130 partners.

[1] D. Englund, D. Fattal, E. Waks, G. Solomon, B. Zhang, T. Nakaoka, Y. Arakawa, Y. Yamamoto, and J. Vučković, Controlling the Spontaneous Emission Rate of Single Quantum Dots in a Two-Dimensional Photonic Crystal, Phys. Rev. Lett. 95, 013904 (2005).

[2] S. Noda, M. Fujita, and T. Asano, Spontaneous-emission control by photonic crystals and nanocavities, Nat. Photonics 1, 449 (2007).

[3] J. P. Reithmaier, G. Sek, A. Löffler, C. Hofmann, S. Kuhn, S. Reitzenstein, L. V. Keldysh, V. D. Kulakovskii, T. L. Reinecke, and A. Forchel, Strong coupling in a single quantum dot-semiconductor microcavity system, Nature (London) 432, 197 (2004).

[4] H. Yuan, S. Khatua, P. Zijlstra, M. Yorulmaz, and M. Orrit, Thousand-fold enhancement of single-molecule fluorescence near a single gold nanorod, Angew. Chem., Int. Ed. Engl. 52, 1217 (2013).

[5] A. Kinkhabwala, Z. Yu, S. Fan, Y. Avlasevich, K. Müllen, and W.E. Moerner, Large single-molecule fluorescence enhancements produced by a bowtie nanoantenna, Nat. Photonics 3, 654 (2009).

[6] J. Wenger, B. Cluzel, J. Dintinger, N. Bonod, A.-L. Fehrembach, E. Popov, P.-F. Lenne, T. W. Ebbesen, and H. Rigneault, Radiative and nonradiative photokinetics alteration inside a single metallic nanometric aperture, J. Phys. Chem. C 111, 11469 (2007). 
[7] M. Agio and A. Alù, Optical Antennas (Cambridge University Press, Cambridge, England, 2013).

[8] H. Gersen, M. F. García-Parajó, L. Novotny, J. A. Veerman, L. Kuipers, and N. F. van Hulst, Influencing the Angular Emission of a Single Molecule, Phys. Rev. Lett. 85, 5312 (2000).

[9] G. Vecchi, V. Giannini, and J. Gómez Rivas, Shaping the Fluorescent Emission by Lattice Resonances in Plasmonic Crystals of Nanoantennas, Phys. Rev. Lett. 102, 146807 (2009).

[10] S. R. K. Rodríguez, S. Murai, M. A. Verschuuren, and J. Gómez Rivas, Light-Emitting Waveguide-Plasmon Polaritons, Phys. Rev. Lett. 109, 166803 (2012).

[11] G. Lozano, D. J. Louwers, S. R. K. Rodríguez, S. Murai, O. T. A. Jansen, M. A. Verschuuren, and J. Gómez Rivas, Plasmonics for solid-state lighting: Enhanced excitation and directional emission of highly efficient light sources, Light Sci. Appl. 2, e66 (2013).

[12] C. Belacel, B. Habert, F. Bigourdan, F. Marquier, J.-P. Hugonin, S. Michaelis de Vasconcellos, X. Lafosse, L. Coolen, C. Schwob, C. Javaux, B. Dubertret, J.-J. Greffet, P. Senellart, and A. Maitre, Controlling spontaneous emission with plasmonic optical patch antennas, Nano Lett. 13, 1516 (2013).

[13] A. Mohtashami, T. Coenen, A. Antoncecchi, A. Polman, and A.F. Koenderink, Nanoscale excitation mapping of plasmonic patch antennas, ACS Photonics 1, 1134 (2014).

[14] Alberto G. Curto, Giorgio Volpe, Tim H. Taminiau, Mark P. Kreuzer, Romain Quidant, and Niek F. van Hulst, Unidirectional emission of a quantum dot coupled to a nanoantenna, Science 329, 930 (2010).

[15] H. J. Lezec, A. Degiron, E. Devaux, R. A. Linke, L. MartínMoreno, F. J. García-Vidal, and T. W. Ebbesen, Beaming light from a subwavelength aperture, Science 297, 820 (2002).

[16] J. Wenger, P.-F. Lenne, E. Popov, H. Rigneault, J. Dintinger, and T. Ebbesen, Single molecule fluorescence in rectangular nano-apertures, Opt. Express 13, 7035 (2005).

[17] J. Wenger, D. Gérard, J. Dintinger, O. Mahboub, N. Bonod, E. Popov, T. W. Ebbesen, and H. Rigneault, Emission and excitation contributions to enhanced single molecule fluorescence by gold nanometric apertures, Opt. Express 16, 3008 (2008).

[18] J. Wenger and H. Rigneault, Photonic methods to enhance fluorescence correlation spectroscopy and single molecule fluorescence detection, Int. J. Mol. Sci. 11, 206 (2010).

[19] O. Mahboub, S. Carretero Palacios, C. Genet, F. J. García-Vidal, Sergio G. Rodrigo, L. Martín-Moreno, and T. W. Ebbesen, Optimization of bull's eye structures for transmission enhancement, Opt. Express 18, 11292 (2010).

[20] H. 1. Aouani, O. Mahboub, N. Bonod, E. Devaux, E. Popov, H. Rigneault, T. W. Ebbesen, and J. Wenger, Bright unidirectional fluorescence emission of molecules in a nanoaperture with plasmonic corrugations, Nano Lett. 11, 637 (2011).

[21] H. Aouani, O. Mahboub, E. Devaux, H. Rigneault, T. W. Ebbesen, and J. Wenger, Plasmonic antennas for directional sorting of fluorescence emission, Nano Lett. 11, 2400 (2011).

[22] I. Sersic, M. A. van de Haar, F. Bernal Arango, and A. F. Koenderink, Ubiquity of Optical Activity in Planar Metamaterial Scatterers, Phys. Rev. Lett. 108, 223903 (2012).
[23] M. Schäferling, D. Dregely, M. Hentschel, and H. Giessen, Tailoring Enhanced Optical Chirality: Design Principles for Chiral Plasmonic Nanostructures, Phys. Rev. X 2, 031010 (2012).

[24] F. Bernal Arango and A.F. Koenderink, Polarizability tensor retrieval for magnetic and plasmonic antenna design, New J. Phys. 15, 073023 (2013).

[25] S. S. Kruk, M. Decker, I. Staude, S. Schlecht, M. Greppmair, D. N. Neshev, and Y. S. Kivshar, Spin-polarized photon emission by resonant multipolar nanoantennas, ACS Photonics 1, 1218 (2014).

[26] J. R. Lakowicz, Principles of Fluorescence Spectroscopy (Springer, New York, 2006).

[27] C. Fallet, T. Novikova, M. Foldyna, S. Manhas, B. H. Ibrahim, A. De Martino, C. Vannuffel, and C. Constancias, Overlay measurements by Mueller polarimetry in back focal plane, J. Micro/Nanolith. MEMS MOEMS 10, 033017 (2011).

[28] O. Arteaga, B. M. Maoz, S. Nichols, G. Markovich, and B. Kahr, Complete polarimetry on the asymmetric transmission through subwavelength hole arrays, Opt. Express 22, 13719 (2014).

[29] C. I. Osorio, A. Mohtashami, and A. F. Koenderink, K-space polarimetry of bullseye plasmon antennas, Sci. Rep. 5, 9966 (2015).

[30] Y. Gorodetski, A. Drezet, C. Genet, and T. W. Ebbesen, Generating Far-Field Orbital Angular Momenta from Near-Field Optical Chirality, Phys. Rev. Lett. 110, 203906 (2013).

[31] M. Born and E. Wolf, Principles of Optics, 7th ed. (Cambridge University Press, Cambridge, England, 1999).

[32] M. Bass, C. DeCusatis, J. Enoch, V. Lakshminarayanan, G. Li, C. Macdonald, V. Mahajan, and E. Van Stryland, Handbook of Optics, 3rd ed. (McGraw-Hill, Inc., New York, 2010), Vol. 1.

[33] H. G. Berry, G. Gabrielse, and A. E. Livingston, Measurement of the stokes parameters of light, Appl. Opt. 16, 3200 (1977).

[34] L. Langguth, D. Punj, J. Wenger, and A. F. Koenderink, Plasmonic band structure controls single molecule fluorescence, ACS Nano 7, 8840 (2013).

[35] P. Kapusta, R. Erdmann, U. Ortmann, and M. Wahl, Time-resolved fluorescence anisotropy measurements made simple, J. Fluoresc. 13, 179 (2003).

[36] M. Ameloot, M. vandeVen, A. U. Acuña, and B. Valeur, Fluorescence anisotropy measurements in solution: Methods and reference materials, Pure Appl. Chem. 85, 589 (2013).

[37] A. Mohtashami and A. F. Koenderink, Suitability of nanodiamond nitrogen-vacancy centers for spontaneous emission control experiments, New J. Phys. 15, 043017 (2013).

[38] L. Novotny and B. Hecht, Principles of Nano-Optics (Cambridge University Press, Cambridge, England, 2006).

[39] S. A. Maier, Plasmonics: Fundamentals and Applications (Springer, New York, 2007)

[40] F. J. Rodríguez-Fortuño, G. Marino, P. Ginzburg, D. O'Connor, A. Martínez, G. A. Wurtz, and A. V. Zayats, Near-field interference for the unidirectional excitation of electromagnetic guided modes, Science 340, 328 (2013). 